\title{
A novel application method for wearable bend sensors
}

\author{
Giovanni Saggio, Stefano Bocchetti, Carlo Alberto Pinto, Giancarlo Orengo, Franco Giannini \\ Dept. of Electronic Engineering \\ University of Rome "Tor Vergata" \\ Via del Politecnico 1, 00133 Rome, Italy \\ saggio@uniroma2.it
}

\begin{abstract}
Bend sensors fundamental characteristic is to furnish an electrical resistance value related to the angle they are bent. This feature can be successfully exploited to realize wearable systems capable to measure human static and dynamic postures. In particular some efforts have been made to determine finger joint movements of human hands and it has been demonstrated the feasibility of using the so called data glove system as a goniometric device. The repeatability of such system is quite good for general purposes but it is still not sufficient for specific applications (for instance in virtual surgery). So here we introduce a novel application method of bend sensors and demonstrate how it can be useful to improve the system repeatability.
\end{abstract}

Keywords-component; bend sensors; sensor array; wearable devices; data glove

\section{INTRODUCTION}

We can take advantage of the bend sensor's characteristics $[1,2]$ to successfully measure the finger joint static and dynamic postures. To this aim each sensor must be bent exactly according to each finger joint movements. The ensemble of bend sensors, a supporting glove, a conditioning electronics, a wired/wireless transmission system, form a so called data glove [3]. Such a glove is capable to virtually measure all the degree of freedom of finger joint movements, so to furnish extremely information-rich data sets extremely important if utilized, for instance, in rehabilitation applications. In fact doctors and therapists can utilize these data to monitor the rehabilitation exercises, to control and verify the exercises off-line, to modify the therapy in real time, to evaluate disabilities, etc. But to enlarge the field of possible applications, especially where the precision is a key element, the data glove repeatability must be improved.

So we propose here a data glove based on bend sensors utilized in a novel configuration. Since the glove has been developed by the Hiteg (Health Involved Technical Engineering Group, http://hiteg.uniroma2.it/) laboratory, was termed HG. Performances of our glove are here compared to a well established one such as the Humanware HumanGlove (HHG) [4], and a state of the art one i.e. the Wuerzburg University Glove (WVG) [5].

\section{Hiteg DATA GLOVE}

As a routine procedure, data gloves are generally realized with one sensor for each joint, in a way that every sensor is fully independent and acts separately from the others. Here we propose a novel configuration for which the three sensors for the three joints of the same finger lie on the same substrate, forming a sensor array, as in fig. 1.

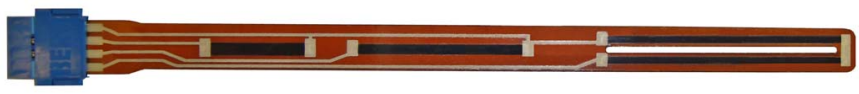

Figure 1. An array of three sensors on the same substrate.

All the contacts are placed on one tip of the array, so to partially solve the problem of disorder due to the connecting wires. The sensor placed on the other tail of the array, in correspondence of the metacarpophalangeal joint, has a middle slot (see fig. 2) so to constrain the array to move always on the same direction with finger flexure.

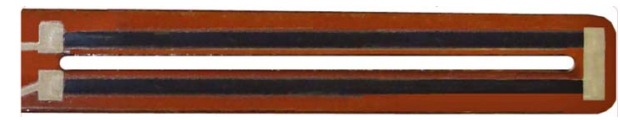

Figure 2. zoom of part of fig. 1.

The fig. 3 shows the arrays placed on the Lycra glove. The arrays slide on the respective finger with hand closure.

We experienced some advantages in utilizing sensors in array configuration:

- The same substrate assures three sensors to be kept always aligned with each other, avoiding sensor misalignment during the glove usage

- The array remains aligned with the respective finger thanks to the designed middle slot

- The electrical contacts are grouped in one tip of the array avoiding wire tangles

- The array assures a single electrical mass for three different sensors, avoiding shift in reference potentials 
- One array design can be easily adopted for all the fingers since it is sufficient to scale the design according to the finger sizes

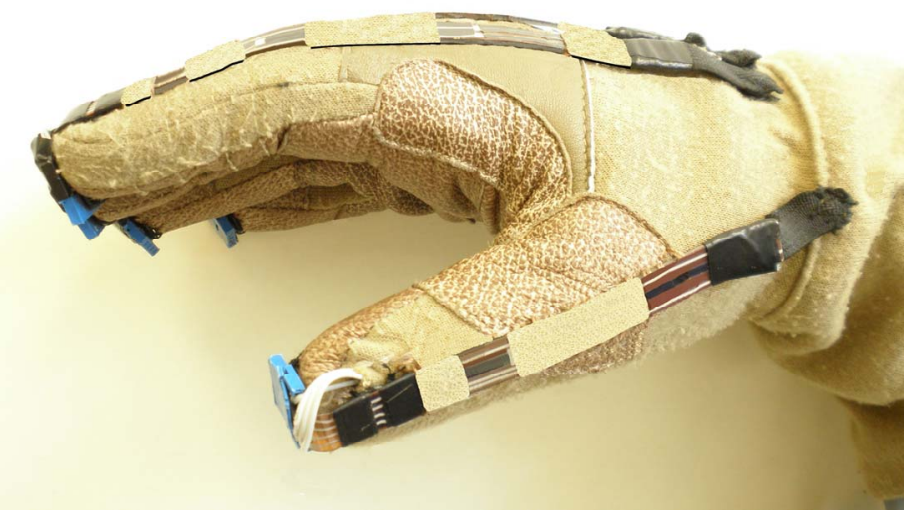

Figure 3. Hiteg data glove

Our aim was then to evaluate the performances of our data glove with respect to two known previously mentioned state of the art data gloves, i.e. the HHG already characterized by Dipietro et al. [4], and the WVG measured by Gentner and Classen [5]. In such a view we adopted a standard measure procedure as in [6], later modified [4], but with some minor differences here introduced to overcome recognized problems. The procedure consists of four testing protocols (tests from $A$ to $D$, later detailed) performed by voluntary subjects, wearing a data glove, and for whom custom plastic mold were realized so that the same grip characteristics were obtained for repeatability testing. The subjects were six healthy individuals, four men and two women, aged 23-29, right-handed, with normal hand functions. The glove was placed on the dominant right hand for all.

\section{EXPERIMENTAL PROCEDURES}

Four different tests were performed on the glove so to evaluate measurement repeatability. The first two (tests $A$ and $B$ ) consisted of gripping a pre-formed mold so to replay a predefined static hand posture, while the second two (tests $C$ and $D)$ were performed so to replay a closed fist position. In details:

\section{A. $\quad$ Test $A$}

The subjects, previously trained, were asked to hold (not to clench) his/her customized mold (see fig. 4a) for $6 \mathrm{~s}$ and to release the mold placing his/her hand in a pre-imposed flat position (see fig. $4 \mathrm{~b}$, forearm in a prone-supine and the wrist in a neutral position) on a desk for additional $6 \mathrm{~s}$. These actions corresponded to 1 trial, for which the $X$-th data is acquired as an average of at least 130 measures. The same actions were cycled 10 times always maintaining the glove donned. The average $\bar{X}$ of all the ten $X$ data forms 1 data block. All the procedures were repeated again 10 times until obtaining 10 data blocks in total (100 trials).

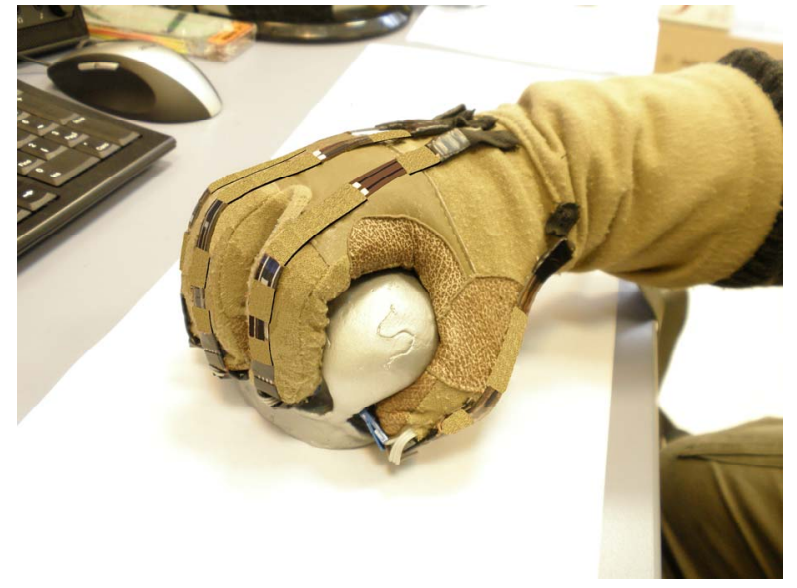

(a)

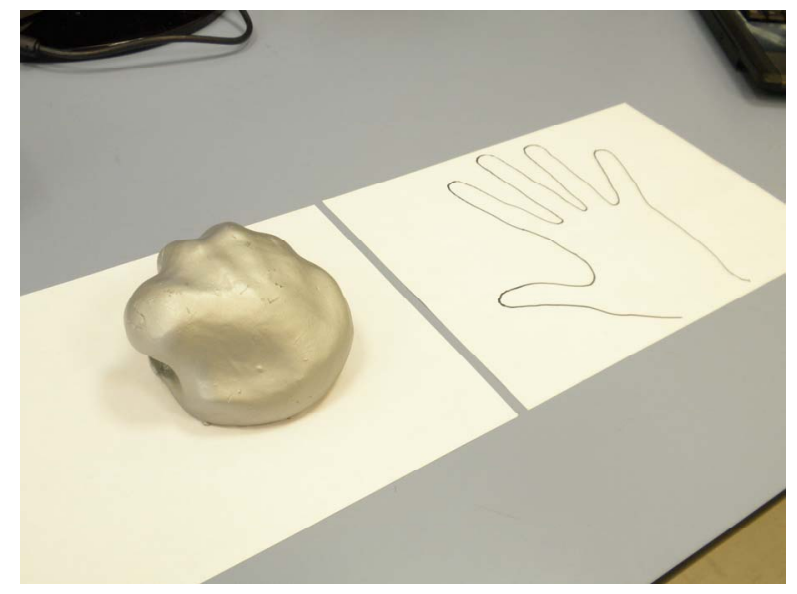

(b)

Figure 4. (a) hand holding a customized mold, (b) prepared test A protocol

\section{B. Test $B$}

The same procedures as in test $A$ with the difference of doffing and donning the glove between each cycle. Presumably the glove did not fit the hand every time exactly in the same position, so this test helped us to evaluate such effect on the measurement process.

\section{Test $C$}

The subjects were asked to clench the hand lightly in maximum flexion position for $6 \mathrm{~s}$ instead of gripping the mold. All the rest was done as a clone of test $A$.

\section{Test D}

The same procedures as test $C$ with the difference of doffing and donning the glove between each cycle, so to evaluate the effects as mentioned for test $B$. 


\section{PROTOCOL DIFFERENCES}

Differing from previously reported testing protocol for which the molds arranged a roughly cylindrical aspect, we chosen to modeled our molds with the aim to assure a comfortable closing hand position. In such a manner it was practically eliminated the problem that changes in grip force affected measured values [4], since no force is necessary to keep the hand on the position imposed by the mold.

\section{RESULTS}

For the $j$-th data block and the $k$-th sensor, we calculated the range $R_{E}=\max _{i}\left(\overline{X_{j k}}\right)-\min _{j}\left(\overline{X_{i k}}\right)$, its average value $\overline{R_{k}}$ and the $\overline{X_{j k}}$ standard deviation $(S D)$ values. The results were automatically obtained thanks to a home-made acquisition software. The acquired data blocks were then automatically converted in the values defined in order to evaluate the glove repeatability.

Stands the obtained measured results reported in tab. 1, our solution was advantageous to improve the performances of the data glove with respect to the references.

\begin{tabular}{|c|c|c|c|c|c|c|c|c|}
\hline \multirow{2}{*}{ Subject } & \multicolumn{2}{|c|}{ Test A } & \multicolumn{2}{c|}{ Test B } & \multicolumn{2}{c|}{ Test C } & \multicolumn{2}{|c|}{ Test D } \\
\cline { 2 - 9 } & Rk & SD & Rk & SD & Rk & SD & Rk & SD \\
\hline 1, Man & 3.77 & 1.41 & 5.04 & 1.55 & 1.02 & 0.34 & 2.49 & 0.42 \\
\hline 2, Man & 2.49 & 1.17 & 6.47 & 1.29 & 2.06 & 0.50 & 2.30 & 0.50 \\
\hline 3, Man & 4.29 & 1.42 & 6.74 & 1.88 & 2.18 & 0.51 & 2.48 & 0.45 \\
\hline 4, Man & 3.75 & 1.51 & 4.40 & 1.54 & 1.12 & 0.68 & 2.09 & 0.53 \\
\hline $\begin{array}{c}\text { Mean } \\
\text { Male }\end{array}$ & 3.58 & 1.38 & 5.66 & 1.56 & 1.60 & 0.51 & 2.34 & 0.48 \\
\hline 5, Female & 4.16 & 1.66 & 5.05 & 1.88 & 2.07 & 0.48 & 2.27 & 0.78 \\
\hline 6, Female & 4.98 & 1.73 & 6.99 & 2.15 & 2.16 & 0.78 & 2.80 & 0.83 \\
\hline $\begin{array}{c}\text { Mean } \\
\text { Female }\end{array}$ & 4.57 & 1.69 & 6.02 & 2.02 & 2.13 & 0.63 & 2.54 & 0.80 \\
\hline $\begin{array}{c}\text { Overall } \\
\text { Mean }\end{array}$ & $\mathbf{3 . 9 1}$ & $\mathbf{1 . 4 8}$ & $\mathbf{5 . 7 8}$ & $\mathbf{1 . 7 2}$ & $\mathbf{1 . 7 7}$ & $\mathbf{0 . 5 5}$ & $\mathbf{2 . 4 0}$ & $\mathbf{0 . 5 8}$ \\
\hline
\end{tabular}

\begin{tabular}{|c|c|c|c|c|c|c|c|c|}
\hline $\begin{array}{c}\text { Dipietro } \\
\text { '03 }\end{array}$ & 7.47 & 2.6 & 9.38 & 2.96 & 3.84 & 1.23 & 5.88 & 1.92 \\
\hline $\begin{array}{c}\text { Gentner } \\
\text { '09 }\end{array}$ & 6.09 & 1.61 & 7.16 & 2.26 & 2.61 & 0.86 & 3.98 & 1.28 \\
\hline
\end{tabular}

Table 1: $R k$ and $S D$ values obtained from the tests

As a further information, in fig. 5 is reported the histogram of average standard deviation $(S D)$ for measurements taken from each sensors, related to flex/extensions of each finger's joints. Again, the comparison demonstrates the improvement obtained with the array configuration.

\section{CONCLUSIONS}

Data glove based on bend sensors forms a system which can be successfully utilized as a goniometric device. Some authors already demonstrated the performances which can be obtained from such a system. Among all the performance values, playing the repeatability one a key rule for some applications, here we propose a novel method useful to improve it. Our results, here compared with literature reference systems, demonstrated a gain in data glove repeatability.

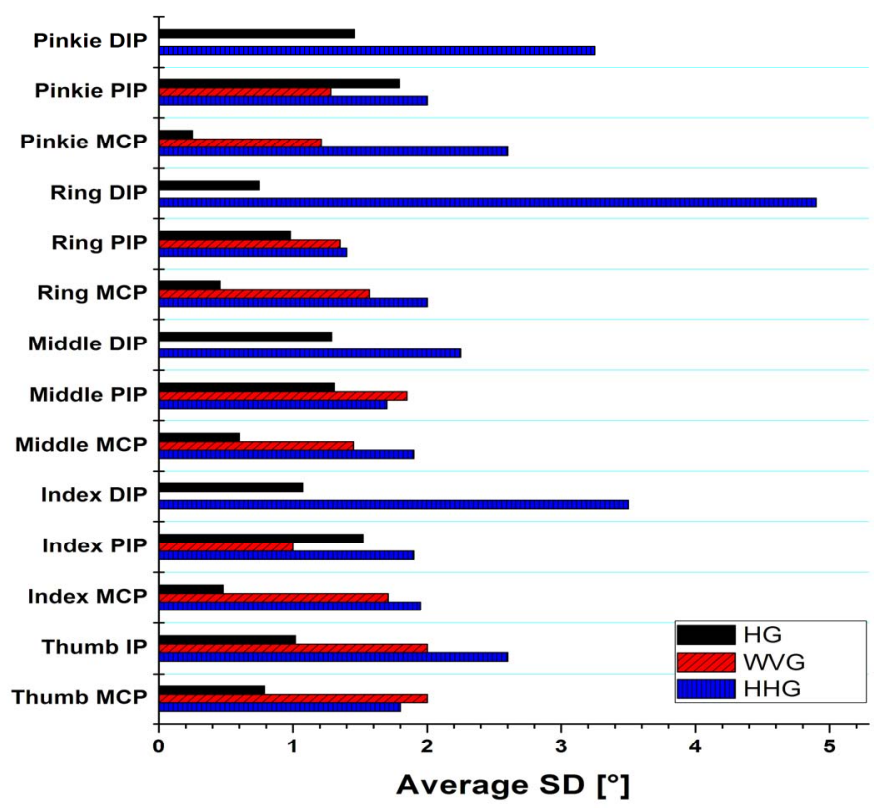

Figure 5. Comparisons of the average SD finger values among three different data gloves (HG, WVG, HHG)

\section{REFERENCES}

[1] G. Saggio, G. Latessa, S. Bocchetti, F. Brunetti, A. Reale, F. Giannini, "Sensor's Design and Characterization for Biomedical Applications," ISSBB, IV National Congress, Santa Margherita Ligure, April 2009

[2] G. Orengo, L. Giovannini, G. Latessa, G. Saggio, F. Giannini, "Characterization of piezoresistive sensors for goniometric glove in hand prostheses," Wireless Vitae09 - Wireless Communications, Vehicular Technology, Information Theory and Aerospace \& Electronic Systems Technology, Aalborg (Denmark), 17-20 May, 2009 - pp. 684687.

[3] L. Dipietro, A. M. Sabatini, P. Dario, "A Survey of Glove-Based Systems and Their Applications," IEEE Transactions on Systems, Man, and Cybernetics_-part C: Applications and Reviews, vol. 38, no. 4, july 2008, pp. 461-482.

[4] L. Dipietro, A. M. Sabatini, P. Dario, "Evaluation of an instrumented glove for hand movement acquisition," J Rehabil Res Dev 2003;40(2):179-90.

[5] R. Gentner, J. Classen, "Development and evaluation of a low-cost sensor glove for assessment of human finger movements in neurophysiological settings," Journal of Neuroscience Methods 178 (2009) 138-147

[6] S. Wise, W. Gardner, E. Sabelman, E. Valainis, Y. Wong, K. Glass, et al., "Evaluation of a fiber optic glove for semi-automated goniometric measurements," J Rehabil Res Dev 1990;27(4):411-24. 